\title{
Processes and mechanisms governing the initiation and propagation of CMEs
}

\author{
B. Vršnak \\ Hvar Observatory, Faculty of Geodesy, Kačićeva 26, 10000 Zagreb, Croatia
}

Received: 25 September 2007 - Revised: 26 November 2007 - Accepted: 26 November 2007 - Published: 15 October 2008

\begin{abstract}
The most important observational characteristics of coronal mass ejections (CMEs) are summarized, emphasizing those aspects which are relevant for testing physical concepts employed to explain the CME take-off and propagation. In particular, the kinematics, scalings, and the CMEflare relationship are stressed. Special attention is paid to 3dimensional (3-D) topology of the magnetic field structures, particularly to aspects related to the concept of semi-toroidal flux-rope anchored at both ends in the dense photosphere and embedded in the coronal magnetic arcade. Observations are compared with physical principles and concepts employed in explaining the CME phenomenon, and implications are discussed. A simple flux-rope model is used to explain various stages of the eruption. The model is able to reproduce all basic observational requirements: stable equilibrium and possible oscillations around equilibrium, metastable state and possible destabilization by an external disturbance, pre-eruptive gradual-rise until loss of equilibrium, possibility of fallback events and failed eruptions, relationship between impulsiveness of the CME acceleration and the source-region size, etc. However, it is shown that the purely ideal MHD process cannot account for highest observed accelerations which can attain values up to $10 \mathrm{~km} \mathrm{~s}^{-2}$. Such accelerations can be achieved if the process of reconnection beneath the erupting flux-rope is included into the model. Essentially, the role of reconnection is in changing the magnetic flux associated with the flux-rope current and supplying "fresh" poloidal magnetic flux to the rope. These effects help sustain the electric current flowing along the flux-rope, and consequently, reinforce and prolong the CME acceleration. The model straightforwardly explains the observed synchronization of the flare impulsive phase and the CME main-acceleration stage, as well as the correlations between various CME and flare parameters.
\end{abstract}

Keywords. Solar physics, astrophysics, and astrnomy (Flares and mass ejection)

\footnotetext{
Correspondence to: B. Vršnak

(bvrsnak@geof.hr)
}

\section{Introduction}

Coupling of the solar differential rotation, convective motions, and magnetic field results in magnetohydrodynamic (MHD) dynamo processes at different scales. Shearing and twisting motions induce electric currents, storing free energy into current-carrying magnetic field structures. A part of this energy is transferred through the solar surface into the corona, where it is partly spent for the coronal heating and partly released in eruptive processes, taking the form of coronal mass ejections (CMEs) and/or solar flares (cf., Priest, 1982).

Although CMEs show a broad variety of morphological, evolutionary, and kinematical characteristics, there are certain "typical" properties common to most of events. Generally, eruptions start with a phase of a slow rise, most often seen in measurements of the associated prominence eruption. In this gradual pre-eruption stage, typical velocity of the eruptive prominence is in the order of $10 \mathrm{~km} \mathrm{~s}^{-1}$ (e.g., Rompolt, 1990). At the same time, the overlying coronal arches have several times larger velocities (e.g., Maričić et al., 2004).

At a certain height, the slowly-rising structure suddenly starts to accelerate. Most often the leading edge of the eruption shows an acceleration in the range of several hundreds of $\mathrm{m} \mathrm{s}^{-2}$ (Vršnak, 2001; Zhang, 2005; Vršnak et al., 2007), but in most impulsive events the acceleration can attain values in the order of $10 \mathrm{~km} \mathrm{~s}^{-2}$ (Vršnak et al., 2007, and references therein). Maximum velocities achieved range from several tens $\mathrm{km} \mathrm{s}^{-1}$, up to more than $2000 \mathrm{~km} \mathrm{~s}^{-1}$ (e.g., Yashiro et al., 2004, and references therein).

After the main acceleration stage, which can last from several minutes to several hours (Zhang et al., 2004; Zhang, 2005; Zhang and Dere, 2006; Vršnak et al., 2007), fast CMEs start to decelerate under the influence of the "aerodynamic" drag (Vršnak et al., 2004b). On the other hand, CMEs slower than the solar wind continue to accelerate until attaining the solar wind speed (e.g., Gopalswamy et al., 2000).

Analyzing carefully the properties of the relationship between the acceleration and velocity, $a(v)$, of several

Published by Copernicus Publications on behalf of the European Geosciences Union. 
thousands CMEs observed in the radial distance range $R=3-$ 30 solar radii, (Vršnak et al., 2004b) revealed that the Lorentz force still acts at these heights. At the heliocentric distance of 10 solar radii it is in the order of $10 \mathrm{~N} \mathrm{~kg}^{-1}$, decreasing with the heliocentric distance roughly as $R^{-1}$. In extreme cases it can be an order of magnitude larger.

The aim of this paper is to confront basic concepts used to explain the $\mathrm{CME} /$ flare eruptions with empirical constraints put forward by observations. In Sect. 2, observational characteristics that are most relevant for the comprehension of processes and mechanisms causing the CME acceleration are summarized. In Sect. 3 basic physical principles and concepts employed in explaining the CME take-off are presented and observational implications are discussed. Following the described principles, a simple 3-dimensional (3-D) model of the semi-toroidal flux-rope is presented in Sect. 4. The results are discussed and conclusions drawn in Sect. 5.

\section{Summary of CME characteristics}

\subsection{The CME structure and scalings}

CMEs frequently show a three-part structure, traditionally called the frontal rim, the dark cavity, and the bright core (cf., Crifo et al., 1983; Sime et al., 1984; Hundhausen, 1987). In this respect, it is worth noting that solar eclipse observations have revealed an analogous prominence/corona structure: the prominence is usually found in a coronal "void" enclosed by the coronal arch that is surmounted by the coronal streamer (Engvold, 1987; Koutchmy et al., 2004, and references therein). Such a prominence-corona structure, sometimes observed prior to the CME take-off also by soft X-ray imaging instruments (e.g., Hudson et al., 1999, and references therein) and white-light coronagraphs, (Gibson, 2008; Burkepile, 2008), indicates that the basic CME morphology has its roots in the pre-eruption magnetic field configuration (Low, 1996, and references therein). Usually, it is supposed that the frontal rim outlines the leading edge of the erupting arcade, whereas the cavity reveals the flux-rope within which the prominence is nested (e.g. Low, 1996; Krall et al., 2001; Filippov and Koutchmy, 2008, and references therein). Indeed, white light coronagraphic observations frequently show patterns consistent with the interpretation of CMEs in terms of flux-rope eruption (e.g., Krall, 2007, and references therein). Another line of evidence can be found in the in situ measurements, showing that interplanetary CMEs (ICMEs) frequently have flux-rope characteristics (so called magnetic clouds; for details see Burlaga, 1988; Lepping et al., 1990). For example, according to (Richardson and Cane, 2004), in solar minimum all ICMEs could be classified as magnetic clouds. As the solar activity enhances and the frequency of ICMEs increases, the percentage of magnetic clouds decreases. There are several effects which could explain such a behavior, e.g., ICME-ICME interactions in the interplane- tary space which makes the internal structure too complex to be identified as a set of flux-ropes (for a discussion we refer to Richardson and Cane, 2004; Gopalswamy, 2006).

Studies addressing the relative kinematics of the three-part structure in the acceleration onset stage are relatively rare, since most often it is difficult to identify and measure the frontal rim during the initiation of a CME. On the other hand, such analyzes (Schmahl and Hildner, 1977; Fisher et al., 1981; Illing and Hundhausen, 1985; Low and Hundhausen, 1987; Plunkett et al., 1997; Dere et al., 1999; Wood et al., 1999; Plunkett et al., 2000; Srivastava et al., 2000; Maričić et al., 2004; Vršnak et al., 2004a), offer an exceptionally important input for the modeling of CME dynamics (cf. Forbes, 2000, and references therein).

The basic outcome of measurements is that the 3-part structure generally expands in a self-similar manner (e.g., Maričić et al., 2004; Vršnak et al., 2004a, and references therein). In the majority of events the acceleration of the leading edge and the prominence are synchronized (Maričić et al., in preparation). However, there are indications that prominence might act as a driver, pushing the cavity and the overlying arch (Burkepille, 2008). Another important aspect is that, on average, sizes of CMEs are roughly proportional to the heliocentric distance at which they are measured (Bothmer and Schwenn, 1998).

(Vršnak et al., 2007) analyzed in detail characteristics of the acceleration stage of the eruption employing a coherent sample of CMEs whose structure was resolved already in the low corona in the EUV and soft X-ray (SXR) images, and whose motion could be traced in the Mauna Loa Mark-IV coronagraph field of view, to join the low-coronal measurements with the observations in the LASCO/SoHO field of view. They found that the acceleration phase duration is inversely proportional to the peak (and average) acceleration (see also Zhang, 2005; Zhang and Dere, 2006), and that the acceleration is stronger if achieved at lower heights (see also Vršnak, 2001). Furthermore, the peak (and average) acceleration is generally larger in eruptions which are initially compact (e.g. fast CMEs launched from active regions) than in eruptions characterized by large initial size (e.g. quiescent prominence eruptions).

(Chen et al., 2006) have inferred that the acceleration peak occurs soon after the height of the erupting structure (semitoroidal flux-rope) exceeds the value of the footpoint halfseparation. That is consistent with observations of a set of eruptive prominences with helical morphology, presented by (Vršnak et al., 1991), who demonstrated that the flux-rope starts to accelerate (if sufficiently twisted) when the height becomes comparable with the footpoint half-separation. The results presented by (Vršnak et al., 1991) also indicate that the acceleration starts at lower heights for more twisted prominences. 


\subsection{CME-flare relationship}

An intriguing aspect of CMEs is their relation to solar flares (e.g., Low, 1996; Forbes, 2000; Švestka, 2001, and references therein). Although in general the association rate is not high (e.g., Kahler, 1992; Gosling, 1993; Harrison, 1995, and references therein), there is obviously a physical connection between these two phenomena, especially between fast CMEs and major flares. In particular, the dynamical flares (traditionally called two-ribbon flares) are by definition related to disruptions of coronal magnetic field structures.

A two-ribbon flare occurs as a consequence of the magnetic field reconnection that takes place in the wake of CME, where the vertically stretched field lines form a vertical current sheet (cf., Priest, 1982). The acceleration phase of the ejection in majority of events starts earlier than the rise of the flare-associated SXR emission. According to (Maričić et al., 2007) the SXR emission can be delayed up to one hour, whereas the average lag equals to $\approx 20 \mathrm{~min}$. Furthermore, signatures of non-thermal electrons (radio and hard $\mathrm{X}$-ray emission) are usually very weak (or even absent) during the gradual pre-eruption phase and at the beginning of the acceleration stage (see, e.g., Kahler et al., 1988; Sterling and Moore, 2004; Vršnak et al., 2003). This shows that dynamical flares occur as a consequence of the eruption, and indicates that the eruption is initially driven by ideal MHD processes.

Various observations indicate that the CME dynamics is closely related to the energy release in the associated flare, or vice versa, the energy release in flare is tightly associated with the CME kinematics. For example, statistical studies show that CME parameters, like the velocity or kinetic energy, are correlated with characteristics of the associated flare, e.g. the SXR peak flux or integrated flux (Moon et al., 2002, 2003; Burkepile et al., 2004; Vršnak et al., 2005). Furthermore, case studies reveal the synchronization of the flare impulsive phase and the CME acceleration stage (e.g., Kahler et al., 1988; Neupert et al., 2001; Zhang et al., 2001; Shanmugaraju et al., 2003; Maričić et al., 2004; Vršnak et al., 2004a; Zhang et al., 2004; Zhang and Dere, 2006; Maričić et al., 2007; Temmer et al., 2007b).

(Maričić et al., 2007) have analyzed in detail the relationship between the acceleration of the eruption and the timeline of energy release in the associated flare employing a coherent sample of well observed CMEs. The main outcome of this study was that in most of events the CME acceleration phase is synchronized with the impulsive phase of the associated flare. In this way the results reported by (Zhang et al., 2001), (Vršnak et al., 2004a), and (Zhang and Dere, 2006) were confirmed from statistical point of view, indicating that reconnection plays a very important role in the CME acceleration. Yet, it should be emphasized that in almost one quarter of events there was practically no synchronization. A similar conclusion was drawn by (Vršnak et al., 2004a) after revisiting a number of previous studies.
In this respect, it is important to note that a certain fraction of ejections is not accompanied by any significant flare energy release. Nevertheless, in such events a growing system of post-eruption loops, similar to that in two-ribbon flares (post-flare loops), is often observed (e.g., Vršnak et al., 2005). This implies that the magnetic field reconnection itself, rather than the flare energy release, affects the dynamics of the eruption. Indeed, measurements of the product of the flare-ribbon expansion velocity and the underlying photospheric magnetic field, representing a proxy for the coronal reconnection rate (Poletto and Kopp, 1986), show a close synchronization of the reconnection rate and the CME acceleration (Wang et al., 2003; Qiu et al., 2004; Jing et al., 2005). Furthermore, the active role of reconnection is documented also by morphological changes within the erupting structure (e.g., Maričić et al., 2004; Vršnak et al., 2004a).

Indirect information on the reconnection below the erupting flux-rope may also be found in the so-called disconnecting events (e.g., Webb and Cliver, 1995; Simnett et al., 1997; Wang et al., 1999; Webb et al., 2003), thin streamer-like coronal features (e.g., Ko et al., 2003; Webb et al., 2003; Lin et al., 2005; Bemporad et al., 2006, and references therein), the horizontal converging coronal flows above the X-ray loops (Yokoyama et al., 2001), etc.

\section{General principles and basic concepts}

\subsection{Energy and forces}

The only resource that can provide a sufficient amount of energy for the CME/flare eruption is the coronal magnetic field (e.g., Forbes, 2000). However, it should be kept in mind that the energy of the potential magnetic field cannot be converted to other forms of energy, i.e. only the non-potential field contains the free-energy (e.g., Régnier and Priest, 2007). This means that the mechanical energy of the eruption can be provided only if the pre-eruption coronal structure carries electric currents.

The magnetic field and current system of the pre-eruptive coronal structure is probably very complex. However, bearing in mind that the magnetic structure is rooted in the photosphere, it is instructive to consider the simplest possible approximation, representing the coronal current system by a simple line-current loop, connected to the solar surface at two footpoints (Fig. 1).

Due to the high conductivity of coronal plasma, the magnetic flux through the area encompassed by the current loop and the solar surface, $\Phi$, can be changed only by the flux emergence or submergence, or by reconnection of coronal fields. This has very important implications for the behavior of the system sketched in Fig. 1b. For example, if the current-carrying loop is pushed downwards, the condition $\Phi=$ const. implies that the magnetic field $B$ between the loop and the surface has to increase. Consequently, the magnetic 


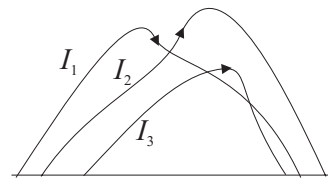

(a)

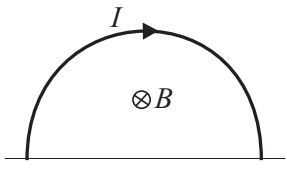

(b)
Fig. 1. Sketch of a complex coronal electric current system (a), and its approximation by the line current (b). The magnetic field $\boldsymbol{B}$ encompassed by the line current and the solar surface is indicated.

pressure increases, giving rise to the restoring force that tends to move the loop back toward its initial (equilibrium) position. Analogously, the restoring force acts downward if the loop is displaced upward. Thus, if the loop would be displaced, it would oscillate around the equilibrium position (for details see, e.g., Vršnak, 1984, 1990). To a certain degree, the effect is analogous to the magnet levitating in a highlyconductive bowl.

This effect was first recognized by (Kuperus and Raadu, 1974), who represented solar prominences by the horizontal line-current $I$ located at the height $h$ above the surface. They expressed the restoring-force effect by employing the mirror current $-I$ located at the depth $h$ below the surface. Hereinafter, we call this force, which is presumably caused by the induced eddy surface currents that prevent the exchange of the magnetic flux through the surface, "the mirror-current effect". The force exerted per unit length of an infinite straight line-current reads $F_{m c}=\frac{\mu_{0} I^{2}}{4 \pi h}$ (Kuperus and Raadu, 1974).

Let us first consider the eruption of the current-carrying structure, which is fast enough, so that the flux $\Phi$ remains approximately constant. Furthermore, let us assume that the current circuit is closed by eddy currents at the solar surface.

In that case, the flux $\Phi$ is related to the current $I$ by the expression $\Phi=L I$, where $L$ is the inductivity of the current circuit. Since the inductivity of the current system is proportional to its size (Jackson, 1998), ${ }^{1}$ the condition $\Phi=$ const. implies that the current has to decrease in the course of the eruption. The inductive decay of the current implies that the Lorentz force decreases, as well as the free energy contained in the system $\left(W=L I^{2} / 2\right)$. The energy is converted into the kinetic and potential energy and to the work done against the "aerodynamic" drag, i.e., the energy carried away by MHD waves (Cargill et al., 1996; Cargill, 2004; Vršnak et al., 2004b).

In the next, somewhat better approximation, we can represent the erupting structure by the flux-rope. The previously described effects would have pretty much the same characteristics, however, now two additional effects appear, due to

\footnotetext{
${ }^{1}$ Generally, the inductivity of a thin circular loop adds up to $L=\frac{\mu_{0} C}{4 \pi}\left[\ln \frac{\xi A}{r^{2}}+\frac{1}{2}\right]$, where $C, A$, and $2 r$ are the circumference, area, and the diameter of the loop, respectively, whereas $\xi$ is a constant in the order of unity (Jackson, 1998, p. 218).
}
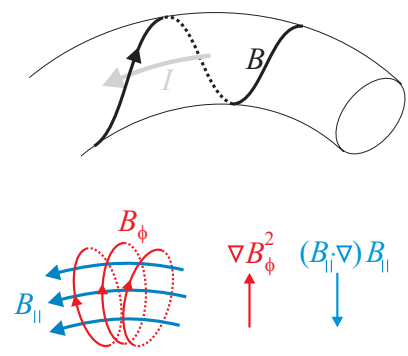

Fig. 2. A curved flux-rope carrying current $I$. At the bottom panel the poloidal $\left(B_{\phi}\right)$ and axial $\left(B_{\|}\right)$magnetic field components are sketched: the gradient of the poloidal field pressure is directed upward, and the tension of the axial component acts downward.

the curvature of the flux-rope (Fig. 2). First, tension of the axial field $B_{\|}$gives rise to the downward force which can be approximately expressed as $F_{t}=\frac{\mu_{0} I^{2}}{4 \pi R X^{2}}\left[\mathrm{~N} \mathrm{~m}^{-1}\right]$, where $X=B_{\phi} / B_{\|}$is the poloidal-to-axial field ratio at the flux-rope surface, and $R$ is the radius of curvature of the flux-rope axis (Vršnak, 1984, 1990). The other effect is the gradient of the poloidal-field pressure ("kink-effect"). In the simplest form it reads $F_{k}=\frac{\mu_{0} I^{2}}{8 \pi R}\left[\mathrm{~N} \mathrm{~m}^{-1}\right]$ (Vršnak, 1984), which is a good approximation for the hoop force derived by applying the principle of virtual work (for details see Žic et al., 2007, and references therein).

Finally, the flux rope is embedded in the background coronal field (sometimes denoted as external poloidal field, strapping field, or overlying field), which has a stabilizing effect to the structure. In the simplest form, it was introduced by (van Tend and Kuperus, 1978), who extended the aforementioned Kuperus-Raadu model by adding the unit-length force $I B_{c}$ due to the background field $B_{c}$.

\subsection{Basic scalings}

Since the eruption is provided by the free energy contained in the magnetic field, the kinetic energy density cannot exceed the magnetic energy density, i.e., $\rho v^{2} / 2 \leq B^{2} / 2 \mu_{0}$, or rewritten, $v^{2} \leq B^{2} / \rho \mu_{0}$. In other words, the CME velocity cannot be larger than the Alfvén velocity within the preeruptive structure, $v \leq v_{\mathrm{A}}$. The majority of CMEs shows the velocity in the range $100-1000 \mathrm{~km} \mathrm{~s}^{-1}$, indicating that the Alfvén speed in the pre-eruptive coronal structures generally ranges within, more or less, an order of magnitude. That is consistent with some other measurements in the height range of one solar radius above the solar surface (see Vršnak et al., 2002, and references therein). At these heights, the inferred values of the Alfvén velocity are found in the range 300 $1500 \mathrm{~km} \mathrm{~s}^{-1}$, corresponding to the ratio 1:5.

An order of magnitude estimate of the CME acceleration can be written as $a=v / t \leq v_{\mathrm{A}} / t_{\mathrm{A}}=v_{\mathrm{A}} /\left(\lambda / v_{\mathrm{A}}\right)=v_{\mathrm{A}}^{2} / \lambda$, where $t_{\mathrm{A}}=\lambda / v_{\mathrm{A}}$ represents the Alfvén travel-time across the erupting structure whose size is denoted by $\lambda$. Another way to 
get this expression is to consider the overall magnetic stress, which can be expressed approximately as $a \leq B^{2} / 2 \mu_{0} \lambda$, again giving $a \leq v_{\mathrm{A}}^{2} / \lambda$. Thus, on average, initially-compact CMEs launched from active regions should have larger accelerations than initially-extended CMEs originating from quiescent regions.

Taking $v_{\mathrm{A}}=1000 \mathrm{~km} \mathrm{~s}^{-1}$ and an initially compact ejection characterized by $\lambda \approx 100 \mathrm{Mm}$, one finds $a \leq 10^{4} \mathrm{~m} \mathrm{~s}^{-2}$. This illustrates that accelerations exceeding $1000 \mathrm{~m} \mathrm{~s}^{-2}$ should not be surprising in compact eruptions launched from active regions. However, the accelerations like those measured in the event of 6 November 1997 (Zhang et al., 2001; Cliver et al., 2004), 10 November 2004 (Williams et al., 2005), or 17 January 2005 (Vršnak et al., 2007) are probably close to the upper limit. On the other hand, considering large quiescent filaments outside active regions where $v_{\mathrm{A}}$ is lower and typical dimensions are larger, one can take, e.g. $v_{\mathrm{A}}=400$ $1000 \mathrm{~km} \mathrm{~s}^{-1}$ and $\lambda \approx 1000 \mathrm{Mm}$, which adds up to $a=100$ $1000 \mathrm{~m} \mathrm{~s}^{-2}$.

The relationship $a \propto \lambda^{-1}$ is consistent with measurements presented by (Vršnak et al., 2007), revealing the inverse proportionality of the peak acceleration and the source-region size. Furthermore, bearing in mind $a=v / t$, one finds that the acceleration phase duration $T_{a c c}$ has to satisfy $T_{a c c} \geq \lambda / v_{\mathrm{A}}$, i.e. compact CMEs should be accelerated more impulsively, again consistent with the results presented by (Vršnak et al., 2007). The co-existence of the relationships $a \propto \lambda^{-1}$ and $T_{a c c} \approx t_{\mathrm{A}} \propto \lambda$, explains straightforwardly the inverse proportionality of $a$ and $T_{a c c}$ demonstrated by (Zhang, 2005), (Zhang and Dere, 2006), and (Vršnak et al., 2007).

In this respect, it is worth noting that the analytical models by (Vršnak, 1990), (Chen and Krall, 2003), and (Kliem and Török, 2006) which treat CMEs as toroidal-field structures, predict that accelerations should be larger in the case of smaller footpoint separation. Moreover, these models predict that the peak acceleration should be attained soon after the height becomes comparable with the footpoint halfseparation. Indeed, an evidence for such a scaling was found by (Vršnak et al., 1991) and (Chen et al., 2006).

\subsection{The role of reconnection}

As stated in Sect. 2.2, the CME take-off is often accompanied by the so-called two-ribbon flare (sometimes also denoted as dynamical flare or eruptive flare). After the arcade takes off, a current sheet is formed below the flux-rope (Fig. 3a), between the field lines anchored at the opposite sides of the photospheric magnetic inversion line (e.g., Forbes, 2000; Cheng et al., 2003; Lin et al., 2004). The two-ribbon flare appears as a consequence of fast magnetic field reconnection that takes place in the current sheet (Vršnak and Skender, 2005). The energy released by the reconnection is transported to the chromosphere by electron beams and thermal conduction, forming there two bright ribbons aligned with the magnetic inversion line (see, e.g. Fig. 1 in Lin et al., 2004

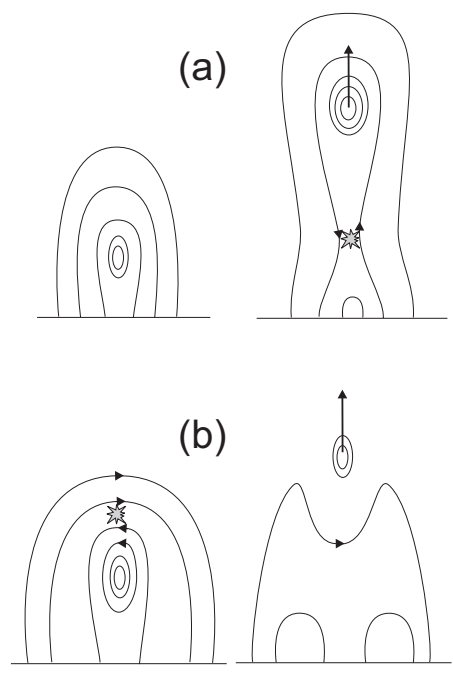

Fig. 3. Schematic drawing of the eruption where reconnection (gray explosion-symbol) takes place: (a) below the flux-rope; (b) above the flux-rope.

or Vršnak and Skender, 2005). As the reconnection proceeds, the ribbons expand away from the inversion line, whereas the reconnected field lines form a growing system of hot X-ray loops (e.g., Vršnak et al., 2004a; Veronig et al., 2006, and references therein).

The described reconnection process has two important effects on the CME. Firstly, the reconnection reduces the net tension of the overlying arcade field and increases the magnetic pressure below the flux-rope, which certainly plays a significant role in the CME dynamics (e.g., van Tend and Kuperus, 1978; Anzer and Pneuman, 1982; Lin, 2002). Secondly, the upward-directed reconnection jet carries the reconnected field lines to the erupting flux-rope, supplying it with a "fresh" poloidal flux. This effect enhances the hoop force, thus reinforcing and prolonging the flux-rope acceleration (Vršnak, 1990). On the other hand, the CME expansion determines the overall geometry of the system and the flows behind the flux-rope, both of which affect the reconnection process (Vršnak and Skender, 2005). In this way, a feedback relationship between the CME motion and the flare energy release is established. The synchronization of the CME acceleration and the energy release in the flare is most likely a consequence of such a feed-back, since the reconnection rate determines also the energy release in the flare (e.g., Asai et al., 2004; Miklenic et al., 2007; Temmer et al., 2007a).

Recently, (Qiu et al., 2007) and Möstl et al. (2008) performed an interesting comparison of the total flux reconnected in the CME-related flares with the magnetic flux of the associated magnetic clouds measured at $1 \mathrm{AU}$. Although based on some ad hoc assumptions, these studies showed that the poloidal magnetic flux of magnetic clouds is comparable with the total magnetic flux reconnected in the parent 

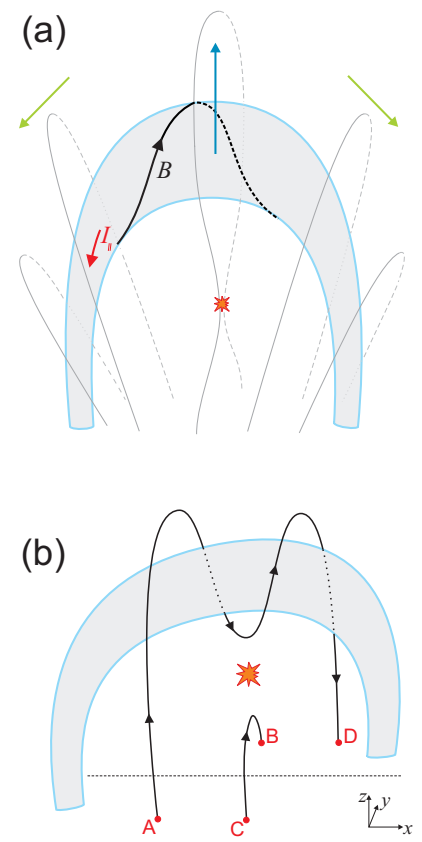

Fig. 4. (a) Schematic drawing of the eruption of a curved fluxrope (light blue) anchored at both ends in the photosphere. The overlying magnetic field is drawn in gray, internal helical field in black, electric current in red. The motion of the flux-rope (deepblue arrow) pushes aside the overlying field (green arrows). The red explosion symbol indicates reconnection below the flux-rope axis. (b) Reconnection of strongly sheared overlying field lines. The magnetic inversion line is indicated by the dashed line oriented in the $x$-direction.

flare/CME process, both being significantly larger than the axial flux of the magnetic cloud. This indicates that a significant part of the magnetic flux of the interplanetary flux-rope is created by reconnection during the ICME take-off.

\section{CME modeling}

\subsection{2-D and 2.5-D models}

There are various forms of 2-D and 2.5-D CME models (cf., Wu et al., 2001; Zhang and Low, 2005; Forbes et al., 2006; Mikić and Lee, 2006, and references therein), and all of them include magnetic reconnection to overcome the problem of the tension of the overlying coronal magnetic field which suppresses the upward acceleration. In the cartesian coordinates the horizontal flux-rope levitates in the core of a horizontal arcade, whose field-lines are anchored at the solar surface. In the spherical geometry the flux-rope encircles the sun, embedded in the magnetic arcade which forms a global helmet streamer. Thus, 2-D and 2.5-D models might be appropriate only to describe the eruption of very long flux-rope/arcade structures, especially in the phase when the height and width of the structure are much smaller than its length.

Depending on the magnetic topology and the location of the reconnection site (Fig. 3), the models could be divided to "tether-cutting" (TC; Moore et al., 1995) and "break-out" (BO; Antiochos, 1998) models. There are various modalities of TC models, but in all of them reconnection takes place below the flux-rope, so the initially overlying magnetic flux is split into the part that wraps around the flux-rope, and a part that forms post-eruption loops. In this respect the standard two-ribbon flare/CME model belongs to the TC class (Fig. 3a). On the other hand, in BO models reconnection takes place above the flux-rope, so the overlying flux is transferred to aside/below the rope (Fig. 3b).

Since in TC models the poloidal flux of the rope increases during the eruption (Fig. 3a), they are consistent with the results reported by (Qiu et al., 2007) and Möstl et al. (2008), who concluded that the poloidal flux of ICMEs is comparable to the flux reconnected in the associated flares (Sect. 3.3). On the other hand, in BO models the poloidal flux decreases during the eruption (Fig. 3b), which seems to contradict the mentioned in situ measurements (however, see also Lynch et al., 2004; Van der Holst et al., 2007). Furthermore, in the case of BO models two sets of growing post-eruption loop systems should be formed on both sides of the main magnetic neutral line located below the flux-rope, and that is not observed. The "main" loop system formed by reconnection below the flux-rope axis (and possibly the two ribbon flare) should appear relatively late, and the footpoints of this loop system (and flare ribbons) should be widely separated. Thus, such model characteristics are not really consistent with observations. Of course, the reconnection above or aside the erupting flux-rope/arcade can take place during the take-off (e.g., Vršnak et al., 1987, 2003) or during propagation in the interplanetary space e.g., Möstl et al. (2008), but it should be considered a consequence of the eruption and not as its primary ingredient.

\subsection{3-D flux-rope model without reconnection}

Unlike in 2-D approach, in 3-D flux-rope models the rope itself is anchored in the photosphere (e.g., Mouschovias and Poland, 1978; Vršnak, 1984; Chen, 1989; Vršnak, 1990; Titov and Démoulin, 1999). Furthermore, since the flux-rope has finite size, the overlying field can be pushed aside by the eruption (Fig. 4a). Thus, reconnection is not necessarily the main mechanism which reduces the tension of the overlying field. In this type of models the effect of moving through the overlying field can be reproduced fairly well by employing the concept of the "aerodynamic" drag (e.g. Cargill et al., 1996; Vršnak et al., 2004b; Cargill, 2004, and references therein). The fast development of numerical MHD techniques in last decade for the first time enabled also the numerical studies of the line-tied flux-rope embedded in the coronal magnetic fields (e.g., Amari et al., 2000, 2003; Roussev et al., 
2003; Kliem et al., 2004; Török and Kliem, 2005; Birn et al., 2006; Gibson et al., 2006, and references therein).

For the matter of simplicity, let us consider the semitoroidal flux-rope whose axis is a circular arc anchored at fixed points in the photosphere, separated by $2 d$ (see Fig. 1 of Vršnak, 1990). In that case the axis length $\Lambda$ and radius of curvature $R$ are simple parametric functions of the height of the axis summit $h$ and the footpoint half-separation $d$. Furthermore, we take the mass density to be uniform within the rope, and the minor radius of the torus $r$ to be constant along the axis (for the effect of variable $r$ see, e.g., Chen and Krall, 2003 , and references therein).

In the absence of reconnection or emerging flux, the poloidal flux of the rope ("internal flux", $\Phi_{i}$ ), as well as the flux encircled by the flux-rope and the solar surface ("external flux", $\Phi_{e}$ ), have to be preserved. For the circular fluxrope axis the internal and external flux behave as $\Phi_{i} \propto I \Lambda$ and $\Phi_{e} \propto I \Lambda\left[\ln \frac{8 R}{r}-2\right]$, respectively (Chen and Krall, 2003; Žic et al., 2007). Thus, in the absence of reconnection or emerging flux ( $\Phi_{i}=$ const. and $\Phi_{e}=$ const.), the current $I$ and the radius $r$ have to obey:

$I \propto \Lambda^{-1}$

and

$r \propto R$.

Equation (2) implies that the rope expands in a self-similar manner, which is one of basic observational results (see Sect. 2.1). The conservation of the poloidal flux also implies (Vršnak, 1990) that the ratio of the poloidal-to-axial field at the flux-rope surface, $X(Z)=B_{\phi} / B_{\|}$, has to behave as:

$X \propto \frac{r}{\Lambda}$.

Under the described approximations, the basic forces acting at the flux-rope summit can be expressed in terms of the geometrical parameters $Z, R$, and $\Lambda$, the flux-rope mass $M$, the axial electric current $I$, and the poloidal-to-axial field ratio $X$ (Vršnak, 1984, 1990). We consider also the force due to the background field $F_{c}=I B_{c}\left[\mathrm{~N} \mathrm{~m}^{-1}\right]$ (Vršnak, 1984). Since the magnetic flux through the vertical cross-section of the flux-rope has to be constant, the background field within the rope has to behave as $B_{c} \propto \Lambda^{-1} r^{-1}$. Finally, the gravitational force should be taken into account, whereas for the matter of simplicity, we neglect the drag term.

Bearing in mind Eq. (1), one can write $\Lambda I=\Lambda_{0} I_{0}$, where $\Lambda_{0}$ and $I_{0}$ are the flux-rope axis length and the electric current at $Z=1$ (the situation when $R=h=d$ ). In the following, the geometrical parameters $Z=h / d, \Lambda, R$, and $r$ are expressed in units of the flux-rope footpoint half-separation $d$, thus $\Lambda_{0} \equiv \pi, Z_{0}=1, R_{0}=1$. Superposing the mentioned forces, the acceleration of the summit flux-rope element can be expressed in the form:

$a=\frac{A}{\Lambda^{2}}\left(\frac{\Lambda}{R}-\frac{2 \Lambda}{R X^{2}}+\frac{2 \Lambda}{Z}\right)+\frac{k_{1}}{r \Lambda^{2}}-k_{2} g(Z)$, where $A=\frac{\pi \mu_{0} I_{0}^{2}}{4 M}$. The three terms in brackets represent the the magnetic pressure gradient of the poloidal field, the tension of the axial field, and the mirror-current effect, respectively (Sect. 3.1). The fourth term represents the $I B_{c}$ force, where the coefficient $k_{1}$ is determined by the background magnetic field strength $B_{c}$ at $Z=1$. In the gravitational term $k_{2} g(Z)$, the buoyancy is taken into account through the coefficient $k_{2}$.

Equations (1-4) determine the dynamics of the eruption. Supplementing the drag term to Eq. (4), and bearing in mind $a=\mathrm{d} v / \mathrm{d} t$, Eq. (4) can be integrated to get the velocity $v(Z)$, from which $Z(t)$ is obtained.

The parameters $X_{0}, A, k_{1}$, and $k_{2}$ determine the height of the flux-rope axis in equilibrium (marked by green dots and denoted as $Z 1$ in Fig. 5a). Different combinations of these parameters result in stable, metastable or unstable equilibria. Stable equilibria are found for low values of $X_{0}$ and $A$. Generally, for a given value of $X_{0}$, the increase of the parameter $A$ shifts the equilibrium to larger heights. If $X_{0}$ is sufficiently large (generally, $X_{0} \gtrsim 2$ ), the system evolves from the stable to a metastable state. The rope remains metastable until $Z_{\text {crit }}$, achieved at $A=A_{\text {crit }}$, where it eventually losses equilibrium and erupts. Such evolution is illustrated in Fig. 5a, where the function $a(Z)$ is shown for $r_{0}=0.1, k_{1}=-517 \mathrm{~m} \mathrm{~s}^{-2}\left(k_{1}<0\right.$ means that the force is directed downward) and $k_{2}=0$ (gravitation neglected, i.e. the coronal and CME densities are equal, $\left.\rho_{\mathrm{c}}=\rho_{\mathrm{CME}}\right)$. The blue curve represents a stable fluxrope, green curves show two metastable situations, whereas the red curve corresponds to the flux-rope that has lost equilibrium ( $a>0$ everywhere). The presented curves demonstrate that with increasing value of $A$ the equilibrium position ( $Z 1$ in Fig. 5a) rises and in the metastable state the $a<0$ region gets successively shallower (green curves in Fig. 5a). Eventually, the dip in the curve becomes tangential with the $x$-axis (red curve in Fig. 5a), marking the state when the equilibrium is lost.

In Fig. $5 \mathrm{~b}$ the function $a(Z)$ is shown for different values of $X_{0}, r_{0}$, and $k_{2}$, using $d=0.1$ solar radii and $k_{1}=1300 \mathrm{~m} \mathrm{~s}^{-2}$. The values of the parameter $A$ are adjusted to the state at which the flux-rope losses equilibrium. The blue and red curves are derived using $k_{2}=0\left(\rho_{\mathrm{c}}=\rho_{\mathrm{CME}}\right)$. Varying the value of $X_{0}$ it is found that loss of equilibrium occurs at somewhat lower heights for larger values of $X_{0}$ (note that the red-dashed curve is displaced to larger heights with respect to the red-full curve). The green curve is obtained by using $k_{2}=1$ (negligible buoyancy, $\rho_{\mathrm{c}} \ll \rho_{\mathrm{CME}}$ ), showing that after a certain height the acceleration becomes negative. This means that there are situations where a stable upper equilibrium exists (denoted as $Z 3$ in Fig. 5b).

Varying the input values over the parameter space, it is found that for physically reasonable values of $X_{0}$ and $A_{0}$, the peak accelerations do not exceed a few hundreds of $\mathrm{m} \mathrm{s}^{-2}$, even assuming strong leakage of mass through the flux-rope legs (Vršnak et al., 1993). In other words, the 3-D flux rope 

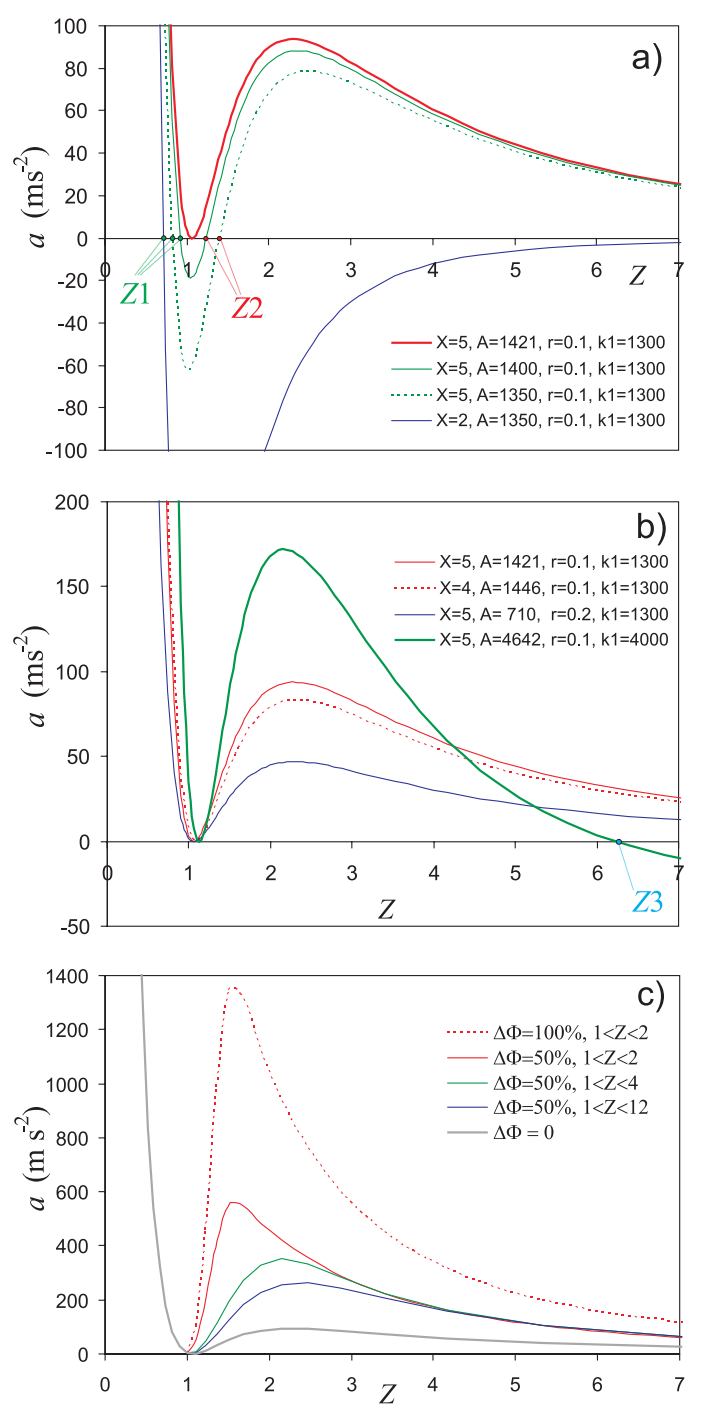

Fig. 5. Characteristics of the function $a(Z)$ : (a) increasing value of $A$ leads to loss of equilibrium (blue - stable flux-rope, green metastable flux-ropes; red - unstable flux-rope); (b) unstable fluxrope for various input parameters (gravity is included only in the case shown by green bold line) (c) the effect of reconnection which supplies the magnetic flux $\Delta \Phi_{e}$; gray line represents the case without reconnection shown in (a) and (b) by the red line $\left(\Delta \Phi_{e}=0\right)$. Parameter values are written in the legend. $Z$ is the height of the flux-rope axis expressed in units of the footpoint half-separation $d$. Stable and metastable equilibria are indicated by green dots (denoted as $Z 1$ ), the unstable equilibria are drawn by red dots $(Z 2)$, and the upper (stable) equilibrium is marked by blue dot $(Z 3)$.

model without reconnection cannot account for the highest observed accelerations, i.e., the flux conservation constraint prevents high accelerations because of the inductive decay of the electric current.

\subsection{3-D flux-rope model with reconnection}

The association of CMEs and two-ribbon flares and growing post-eruption loop systems evidences that reconnection is an essential ingredient of the eruption. The reconnection below the flux-rope axis increases the poloidal flux of the flux-rope and increases the magnetic flux encompassed by the flux-rope and the solar surface - the effect of reconnection is sketched in Fig. 4b, considering the case of a strongly sheared arcade. The sheared field lines, initially connecting $\mathrm{A}$ with $\mathrm{B}$ and $\mathrm{C}$ with $\mathrm{D}$, after reconnection map from $\mathrm{A}$ to $\mathrm{D}$ and from $\mathrm{C}$ to $\mathrm{B}$. The field line $\mathrm{CB}$ represents the postflare loop system. The AD field line is twisted around the rope, meaning that it contributes to the flux-rope current. It should be emphasized that only the $x$-component of the magnetic field (that is the guiding field in reconnection, since it is parallel to the neutral line) contributes to the increase of the magnetic flux $\Phi_{e}$ encircled by the flux-rope and the surface, since in the $y$-direction the reconnection by definition adds equal amount of positive and negative flux. In the case of non-sheared overlying field there is no change of the flux through the circuit, nor is there amendment of the current to the flux-rope (there is no helical field added, i.e. the added poloidal flux around the rope is purely potential. Thus, reconnection in a strongly sheared arcade should support the eruption more (i.e., should provide stronger acceleration) than in a weakly sheared one.

In the following we focus only on the effect of increasing magnetic flux encircled by the flux-rope and the solar surface $\Phi_{e}$. In Fig. 5c the effect of the magnetic field reconnection, causing an increase of $\Phi_{e}$, is shown. Note that, since now $\Phi_{e}$ is not constant, the condition $r / R=$ const. is not necessarily strictly satisfied anymore. Yet, we chose the option of the self-similar expansion, which was necessary condition in the $\Phi_{e}=$ const. case. In such a situation the current has to behave as $I \propto \Phi_{e} \Lambda^{-1}$ (instead of $I \propto \Lambda^{-1}$ valid in the absence of reconnection). Due to the choice $r \propto R$, the behavior of the parameter $X$ remains the same as in the non-reconnection case.

The curves $a(Z)$ shown in Fig. 5c are derived by assuming an increase of the flux $\Phi_{e}$ over a certain height range (indicated in the legend), defined by the function of the type $\Delta \Phi_{e} \propto \cos ^{2} \theta$. In all cases, the values $d=0.1$ solar radii, $X_{0}=5, r_{0}=0.1 k_{1}=1300 \mathrm{~m} \mathrm{~s}^{-2}$, and $k_{2}=0$ are applied, corresponding to the red curve in Figs. $5 \mathrm{a}$ and $\mathrm{b}$. The parameter $A$ is adjusted to the state when the flux-rope losses equilibrium $\left(A=A_{\text {crit }}=1421 \mathrm{~m} \mathrm{~s}^{-2}\right)$.

The two red curves represent the flux increase over the same height range $(1<Z<2)$, but of different amount ( $\Delta \Phi_{e}=50 \%$ and $100 \%$ of the initial flux, respectively). The green and blue curve are both calculated for the flux increase of $\Delta \Phi_{e}=50 \%$, but over the height range $1<Z<4$ and $1<Z<12$ (corresponding to $0.1<h<0.4$ and $0.1<h<1.2$ solar radii, respectively). The gray curve represents the case without reconnection, i.e., the one shown in Fig. $5 a$ and $b$ by 
the red curve $\left(\Delta \Phi_{e}=0\right)$. Obviously, the reconnection significantly enhances the flux-rope acceleration - now accelerations in excess of $1000 \mathrm{~m} \mathrm{~s}^{-2}$ can be achieved easily. Even the highest observed accelerations, in the order of $10 \mathrm{~km} \mathrm{~s}^{-2}$, can be attained if the flux increases by, e.g., $\approx 300 \%$ over the height range $1<Z<2$. Bearing in mind the results by (Qiu et al., 2007) and Möstl et al. (2008), such an increase does not contradict the observations.

With reconnection taking place over an extended period, the acceleration can be prolonged to large heights. For example, taking $d=0.2$ solar radii (corresponding to the footpoint separation of $280 \mathrm{Mm}$ ) and $\Delta \Phi_{e}=50 \%$ provided by reconnection taking place over the height range of $1<Z<12$, one finds the acceleration of about $5 \mathrm{~m} \mathrm{~s}^{-2}$ at the radial distance of 10 solar radii $(Z=45)$, consistent with values obtained by (Vršnak et al., 2004b) at this height range.

\section{Discussion and conclusions}

Some of general characteristics of CMEs described in Sect. 2.1 can be explained already by employing only the most basic physical concepts. For example, it is shown that the peak acceleration and velocity, as well as the acceleration time are determined by the initial source-region size and the magnetic field strength involved. This explains why initially compact eruptions launched from active regions tend to accelerate more impulsively and to attain higher velocities. Finally, the decrease of the Lorentz force with height is straightforwardly explained by the inductive decay of the expanding electric current system.

A more detailed consideration, employing the toroidal flux-rope concept, explains some more detailed characteristics. First of all, the interplay of the mirror-current force, tension force, hoop force, and the Lorentz force associated with the background coronal field can explain existence of stable and metastable structures, as well as the gradual evolution until loss of equilibrium and eruption. Stable structures should react to external disturbances (e.g., coronal shock waves from distant flares or CMEs) by oscillating around the equilibrium, observed as e.g., winking filaments (Ramsey and Smith, 1966). Similar oscillations should occur in the metastable situation if the displacement is not too large. However, if the structure is displaced up to the unstable equilibrium position (denoted in Fig. 5a as Z2), it should erupt. This explains destabilization and eruption of filaments after arrival of coronal shock waves (the phenomenon recognized already by Dodson, 1949).

Generally, an increase of the electric current or increase of the magnetic flux encompassed by the current, drives the evolution of the structure from stable to metastable state. At the same time, the equilibrium height of the structure increases, which explains the slow rising motion usually observed preceding the eruption. At a certain point, usually when the height is comparable with the footpoint half-separation, the system cannot find a neighboring equilibrium state, i.e., the equilibrium is lost and the acceleration phase starts. Model results show that the critical height is lower for more twisted structures, which is consistent with the results presented by (Vršnak et al., 1991).

Modeling of the flux-rope eruption in the frame of ideal MHD (no reconnection) can explain moderate accelerations, up to a few hundreds of $\mathrm{ms}^{-2}$. It also explains the selfsimilar expansion of the CME. Furthermore, for a certain combination of the parameters describing the initial fluxrope, the model shows existence of an upper equilibrium (denoted as $Z 3$ in Fig. 5b). Bearing in mind the damping effect of the aerodynamic drag (Vršnak et al., 1990), this can explain fallback events (Wang and Sheeley, 2002), failed eruptions (Török and Kliem, 2005), and damped oscillations at the upper equilibrium (Vršnak et al., 1990).

However, to explain the highest measured accelerations, which could be as high as $\approx 10 \mathrm{~km} \mathrm{~s}^{-2}$, the flux-rope model has to be extended by inclusion of reconnection, taking place beneath the rising rope. A higher reconnection rate implies a stronger acceleration, which explains correlations between various flare and CME parameters. Furthermore, it is consistent with the synchronization of the CME acceleration and the flare impulsive phase. Finally, it explains in situ measurements of the magnetic fluxes of ICMEs, indicating that significant part of the flux is added to the rope in the course of the eruption.

Acknowledgements. I would like to express my gratitude to the SOC of SOHO-20 who kindly offered me the possibility to present invited talk at the Ghent meeting, and to the LOC of SOHO-20 for warm hospitality and perfect organization of the conference. The team meetings held in the frame of the project "Current Sheet Observations vs. Current Sheet Models" (G. Poletto), organized and sponsored by the International Space Science Institute (ISSI) in Bern, helped me very much in preparing this work. I would also like to thank A. Vourlidas for motivating me to include general considerations presented in Sects. 3.1. and 3.2.

Topical Editor R. Forsyth thanks B. Kliem and J. Raymond for their help in evaluating this paper.

\section{References}

Amari, T., Luciani, J. F., Mikic, Z., and Linker, J.: A Twisted Flux Rope Model for Coronal Mass Ejections and Two-Ribbon Flares, Astrophys. J. Lett., 529, L49-L52, doi:10.1086/312444, 2000.

Amari, T., Luciani, J. F., Aly, J. J., Mikic, Z., and Linker, J.: Coronal Mass Ejection: Initiation, Magnetic Helicity, and Flux Ropes. I. Boundary Motion-driven Evolution, Astrophys. J., 585, 10731086, doi:10.1086/345501, 2003.

Antiochos, S. K.: The Magnetic Topology of Solar Eruptions, Astrophys. J. Lett., 502, L181-L184, doi:10.1086/311507, 1998.

Anzer, U. and Pneuman, G. W.: Magnetic reconnection and coronal transients, Sol. Phys., 79, 129-147, 1982.

Asai, A., Yokoyama, T., Shimojo, M., Masuda, S., Kurokawa, H., and Shibata, K.: Flare Ribbon Expansion and Energy Release Rate, Astrophys. J., 611, 557-567, doi:10.1086/422159, 2004. 
Bemporad, A., Poletto, G., Suess, S. T., Ko, Y.-K., Schwadron, N. A., Elliott, H. A., and Raymond, J. C.: Current Sheet Evolution in the Aftermath of a CME Event, Astrophys. J., 638, 11101128, doi:10.1086/497529, 2006.

Birn, J., Forbes, T. G., and Hesse, M.: Stability and Dynamic Evolution of Three-dimensional Flux Ropes, Astrophys. J., 645, 732741, doi:10.1086/504280, 2006.

Bothmer, V. and Schwenn, R.: The structure and origin of magnetic clouds in the solar wind, Ann. Geophys., 16, 1-24, 1998.

Burkepile, J. T., Hundhausen, A. J., Stanger, A. L., St. Cyr, O. C., and Seiden, J. A.: Role of projection effects on solar coronal mass ejection properties: 1 . A study of CMEs associated with limb activity, J. Geophys. Res., 109, doi:10.1029/2003JA010149, 2004.

Burkepile, J. T., St. Cyr, O. C., Stanger, A. L., et al.: Coronal Mass Ejection accelerations, Ann. Geophys., in preparation, 2008.

Burlaga, L. F.: Magnetic clouds and force-free fields with constant alpha, J. Geophys. Res., 93, 7217-7224, 1988.

Cargill, P. J.: On the Aerodynamic Drag Force Acting on Interplanetary Coronal Mass Ejections, Sol. Phys., 221, 135-149, doi: 10.1023/B:SOLA.0000033366.10725.a2, 2004.

Cargill, P. J., Chen, J., Spicer, D. S., and Zalesak, S. T.: Magnetohydrodynamic simulations of the motion of magnetic flux tubes through a magnetized plasma, J. Geophys. Res., 101, 4855-4870, doi:10.1029/1995JA03769, 1996.

Chen, J.: Effects of toroidal forces in current loops embedded in a background plasma, Astrophys. J., 338, 453-470, doi:10.1086/ 167211, 1989.

Chen, J. and Krall, J.: Acceleration of coronal mass ejections, J. Geophys. Res., 108, 1410, doi:10.1029/2003JA009849, 2003.

Chen, J., Marqué, C., Vourlidas, A., Krall, J., and Schuck, P. W.: The Flux-Rope Scaling of the Acceleration of Coronal Mass Ejections and Eruptive Prominences, Astrophys. J., 649, 452463, doi:10.1086/506466, 2006.

Cheng, C. Z., Ren, Y., Choe, G. S., and Moon, Y.-J.: Flux Rope Acceleration and Enhanced Magnetic Reconnection Rate, Astrophys. J., 596, 1341-1346, doi:10.1086/378170, 2003.

Cliver, E. W., Nitta, N. V., Thompson, B. J., and Zhang, J.: Coronal Shocks of November 1997 Revisited: The Cme Type II Timing Problem, Sol. Phys., 225, 105-139, doi:10.1007/ s11207-004-3258-1, 2004.

Crifo, F., Picat, J. P., and Cailloux, M.: Coronal transients - Loop or bubble, Sol. Phys., 83, 143-152, 1983.

Dere, K. P., Brueckner, G. E., Howard, R. A., Michels, D. J., and Delaboudiniere, J. P.: LASCO and EIT Observations of Helical Structure in Coronal Mass Ejections, Astrophys. J., 516, 465474, doi:10.1086/307101, 1999.

Dodson, H. W.: Position and Development of the Solar Flares of may 8 and 10, 1949., Astrophys. J., 110, 382-386, 1949.

Engvold, O.: Prominence Environment, in: Dynamics and Structure of Solar Prominences, 47-76, 1987.

Filippov, B. and Koutchmy, S.: Causal relationships between eruptive prominences and coronal mass ejections, Ann. Geophys., 26, 3025-3031, 2008, http://www.ann-geophys.net/26/3025/2008/.

Fisher, R., Garcia, C. J., and Seagraves, P.: On the coronal transienteruptive prominence of 1980 August 5, Astrophys. J. Lett., 246, L161-L164, doi:10.1086/183575, 1981.

Forbes, T. G.: A review on the genesis of coronal mass ejec- tions, J. Geophys. Res., 105, 23 153-23 166, doi:10.1029/ 2000JA000005, 2000.

Forbes, T. G., Linker, J. A., Chen, J., Cid, C., Kóta, J., Lee, M. A., Mann, G., Mikić, Z., Potgieter, M. S., Schmidt, J. M., Siscoe, G. L., Vainio, R., Antiochos, S. K., and Riley, P.: CME Theory and Models, Space Sci. Rev., 123, 251-302, doi: 10.1007/s11214-006-9019-8, 2006.

Gibson, S. E., Fan, Y., Török, T., and Kliem, B.: The Evolving Sigmoid: Evidence for Magnetic Flux Ropes in the Corona Before, During, and After CMES, Space Sci. Rev., 124, 131-144, doi:10.1007/s11214-006-9101-2, 2006.

Gibson, S., Fuller, J., Burkepile, J. T., et al.: Coronal filament cavities: why there is a there there and what to do about it, Ann. Geophys., in preparation, 2008.

Gopalswamy, N.: Properties of Interplanetary Coronal Mass Ejections, Space Sci. Rev., 124, 145-168, doi:10.1007/ s11214-006-9102-1, 2006.

Gopalswamy, N., Lara, A., Lepping, R. P., Kaiser, M. L., Berdichevsky, D., and St. Cyr, O. C.: Interplanetary Acceleration of Coronal Mass Ejections, Geophys. Res. Lett., 27, 145148, 2000.

Gosling, J. T.: The solar flare myth, J. Geophys. Res., 98, 1893718 950, doi:10.1029/1993JA01896, 1993.

Harrison, R. A.: The nature of solar flares associated with coronal mass ejection., Astron. Astrophys., 304, 585-594, 1995.

Hudson, H. S., Acton, L. W., Harvey, K. L., and McKenzie, D. E.: A Stable Filament Cavity with a Hot Core, Astrophys. J. Lett., 513, L83-L86, doi:10.1086/311892, 1999.

Hundhausen, A. J.: The Origin and Propagation of Coronal Mass Ejections (R), in: Sixth International Solar Wind Conference, edited by Pizzo, V. J., Holzer, T., and Sime, D. G., 181-214, 1987.

Illing, R. M. E. and Hundhausen, A. J.: Observation of a coronal transient from 1.2 to 6 solar radii, J. Geophys. Res., 90, 275282, 1985.

Jackson, J. D.: Classical Electrodynamics, 3rd Edition, Classical Electrodynamics, 3rd Edition, by John David Jackson, 832 pp., ISBN 0-471-30932-X. Wiley-VCH , July 1998.

Jing, J., Qiu, J., Lin, J., Qu, M., Xu, Y., and Wang, H.: Magnetic Reconnection Rate and Flux-Rope Acceleration of Two-Ribbon Flares, Astrophys. J., 620, 1085-1091, doi:10.1086/427165, 2005.

Kahler, S. W.: Solar flares and coronal mass ejections, Ann. Rev. Astron. Astrophys., 30, 113-141, doi:10.1146/annurev.aa. 30.090192.000553, 1992.

Kahler, S. W., Moore, R. L., Kane, S. R., and Zirin, H.: Filament eruptions and the impulsive phase of solar flares, Astrophys. J., 328, 824-829, doi:10.1086/166340, 1988.

Kliem, B. and Török, T.: Torus Instability, Physical Review Letters, 96, 255 002-1-4, doi:10.1103/PhysRevLett.96.255002, 2006.

Kliem, B., Titov, V. S., and Török, T.: Formation of current sheets and sigmoidal structure by the kink instability of a magnetic loop, Astron. Astrophys., 413, L23-L26, doi:10.1051/ 0004-6361:20031690, 2004.

Ko, Y.-K., Raymond, J. C., Lin, J., Lawrence, G., Li, J., and Fludra, A.: Dynamical and Physical Properties of a Post-Coronal Mass Ejection Current Sheet, Astrophys. J., 594, 1068-1084, doi:10. 1086/376982, 2003.

Koutchmy, S., Baudin, F., Bocchialini, K., Daniel, J.-Y., Delabou- 
dinière, J.-P., Golub, L., Lamy, P., and Adjabshirizadeh, A.: The August 11th, 1999 CME, Astron. Astrophys., 420, 709-718, doi: 10.1051/0004-6361:20040109, 2004.

Krall, J.: Are All Coronal Mass Ejections Hollow Flux Ropes?, Astrophys. J., 657, 559-566, doi:10.1086/510191, 2007.

Krall, J., Chen, J., Duffin, R. T., Howard, R. A., and Thompson, B. J.: Erupting Solar Magnetic Flux Ropes: Theory and Observation, Astrophys. J., 562, 1045-1057, doi:10.1086/323844, 2001.

Kuperus, M. and Raadu, M. A.: The Support of Prominences Formed in Neutral Sheets, Astron. Astrophys., 31, 189-193, 1974.

Lepping, R. P., Burlaga, L. F., and Jones, J. A.: Magnetic field structure of interplanetary magnetic clouds at $1 \mathrm{AU}$, J. Geophys. Res., 95, 11 957-11 965, 1990.

Lin, J.: Energetics and Propagation of Coronal Mass Ejections in Different Plasma Environments, Chinese J. Astron. Ast., 2, 539556, 2002.

Lin, J., Raymond, J. C., and van Ballegooijen, A. A.: The Role of Magnetic Reconnection in the Observable Features of Solar Eruptions, Astrophys. J., 602, 422-435, doi:10.1086/380900, 2004.

Lin, J., Ko, Y.-K., Sui, L., Raymond, J. C., Stenborg, G. A., Jiang, Y., Zhao, S., and Mancuso, S.: Direct Observations of the Magnetic Reconnection Site of an Eruption on 2003 November 18, Astrophys. J., 622, 1251-1264, doi:10.1086/428110, 2005.

Low, B. C.: Solar Activity and the Corona, Sol. Phys., 167, $217-$ 265, 1996.

Low, B. C. and Hundhausen, A. J.: The velocity field of a coronal mass ejection - The event of September 1, 1980, J. Geophys. Res., 92, 2221-2230, 1987.

Lynch, B. J., Antiochos, S. K., MacNeice, P. J., Zurbuchen, T. H., and Fisk, L. A.: Observable Properties of the Breakout Model for Coronal Mass Ejections, Astrophys. J., 617, 589-599, doi: 10.1086/424564, 2004.

Maričić, D., Vršnak, B., Stanger, A. L., and Veronig, A.: Coronal Mass Ejection of 15 May 2001: I. Evolution of Morphological Features of the Eruption, Sol. Phys., 225, 337-353, doi:10.1007/ s11207-004-3748-1, 2004.

Maričić, D., Vršnak, B., Stanger, A. L., Veronig, A. M., Temmer, M., and Roša, D.: Acceleration Phase of Coronal Mass Ejections: II. Synchronization of the Energy Release in the Associated Flare, Sol. Phys., 241, 99-112, doi:10.1007/ s11207-007-0291-x, 2007.

Mikić, Z. and Lee, M. A.: An Introduction to Theory and Models of CMEs, Shocks, and Solar Energetic Particles, Space Sci. Rev., 123, 57-80, doi:10.1007/s11214-006-9012-2, 2006.

Miklenic, C. H., Veronig, A. M., Vršnak, B., and Hanslmeier, A.: Reconnection and energy release rates in a two-ribbon flare, Astron. Astrophys., 461, 697-706, doi:10.1051/0004-6361: 20065751, 2007.

Moon, Y.-J., Choe, G. S., Wang, H., Park, Y. D., Gopalswamy, N., Yang, G., and Yashiro, S.: A Statistical Study of Two Classes of Coronal Mass Ejections, Astrophys. J., 581, 694-702, doi: 10.1086/344088, 2002.

Moon, Y.-J., Choe, G. S., Wang, H., Park, Y. D., and Cheng, C. Z.: Relationship Between CME Kinematics and Flare Strength, J. Korean Astronomical Society, 36, 61-66, 2003.

Moore, R. L., Larosa, T. N., and Orwig, L. E.: The wall of reconnection-driven magnetohydrodynamic turbulence in a large solar flare, Astrophys. J., 438, 985-966, doi:10.1086/175140, 1995.

Möstl, C., Miklenic, C., Farrugia, C. J., Temmer, M., Veronig, A., Galvin, A. B., Vršnak, B., and Biernat, H. K.: Two-spacecraft reconstruction of a magnetic cloud and comparison to its solar source, Ann. Geophys., this issue, 2008.

Mouschovias, T. C. and Poland, A. I.: Expansion and broadening of coronal loop transients - A theoretical explanation, Astrophys. J., 220, 675-682, doi:10.1086/155951, 1978.

Neupert, W. M., Thompson, B. J., Gurman, J. B., and Plunkett, S. P.: Eruption and acceleration of flare-associated coronal mass ejection loops in the low corona, J. Geophys. Res., 106, 25215 25 226, doi:10.1029/2000JA004012, 2001.

Plunkett, S. P., Brueckner, G. E., Dere, K. P., Howard, R. A., Koomen, M. J., Korendyke, C. M., Michels, D. J., Moses, J. D., Moulton, N. E., Paswaters, S. E., St. Cyr, O. C., Socker, D. G., Wang, D., Simnett, G. M., Bedford, D. K., Biesecker, D. A., Eyles, C. J., Tappin, S. J., Schwenn, R., Lamy, P. L., and Llebaria, A.: The Relationship of Green-Line Transients to WhiteLight Coronal Mass Ejections, Sol. Phys., 175, 699-718, doi: 10.1023/A:1004981125702, 1997.

Plunkett, S. P., Vourlidas, A., Šimberová, S., Karlický, M., Kotrč, P., Heinzel, P., Kupryakov, Y. A., Guo, W. P., and Wu, S. T.: Simultaneous SOHO and Ground-Based Observations of a Large Eruptive Prominence and Coronal Mass Ejection, Sol. Phys., 194, 371-391, 2000.

Poletto, G. and Kopp, R. A.: Macroscopic electric fields during tworibbon flares., in: The lower atmosphere of solar flares, edited by: Neidig, D. F. (Sunspot:NSO), 453-465, 1986.

Priest, E. R.: Solar magneto-hydrodynamics, Dordrecht, Holland, Boston, D. Reidel Pub. Co. ; Hingham,, 1982.

Qiu, J., Wang, H., Cheng, C. Z., and Gary, D. E.: Magnetic Reconnection and Mass Acceleration in Flare-Coronal Mass Ejection Events, Astrophys. J., 604, 900-905, doi:10.1086/382122, 2004.

Qiu, J., Hu, Q., Howard, T. A., and Yurchyshyn, V. B.: On the Magnetic Flux Budget in Low-Corona Magnetic Reconnection and Interplanetary Coronal Mass Ejections, Astrophys. J., 659, 758-772, doi:10.1086/512060, 2007.

Ramsey, H. E. and Smith, S. F.: Flare-initiated filamei it oscillations, Astron. J., 71, 197-199, 1966.

Régnier, S. and Priest, E. R.: Nonlinear force-free models for the solar corona. I. Two active regions with very different structure, Astron. Astrophys., 468, 701-709, doi:10.1051/0004-6361: 20077318, 2007.

Richardson, I. G. and Cane, H. V.: The fraction of interplanetary coronal mass ejections that are magnetic clouds: Evidence for a solar cycle variation, Geophys. Res. Lett., 31, L18 804-1-4, doi: 10.1029/2004GL020958, 2004.

Rompolt, B.: Small Scale Structure and Dynamics of Prominences, Hvar Observatory Bulletin, 14, 37-102, 1990.

Roussev, I. I., Gombosi, T. I., Sokolov, I. V., Velli, M., Manchester, IV, W., DeZeeuw, D. L., Liewer, P., Tóth, G., and Luhmann, J.: A Three-dimensional Model of the Solar Wind Incorporating Solar Magnetogram Observations, Astrophys. J. Lett., 595, L57-L61, doi:10.1086/378878, 2003.

Schmahl, E. and Hildner, E.: Coronal mass-ejections-kinematics of the 19 December 1973 event, Sol. Phys., 55, 473-490, 1977.

Shanmugaraju, A., Moon, Y.-J., Dryer, M., and Umapathy, S.: On 
the kinematic evolution of flare-associated cmes, Sol. Phys., 215, 185-201, 2003.

Sime, D. G., MacQueen, R. M., and Hundhausen, A. J.: Density distribution in looplike coronal transients - A comparison of observations and a theoretical model, J. Geophys. Res., 89, 21132121, 1984.

Simnett, G. M., Tappin, S. J., Plunkett, S. P., Bedford, D. K., Eyles, C. J., St. Cyr, O. C., Howard, R. A., Brueckner, G. E., Michels, D. J., Moses, J. D., Socker, D., Dere, K. P., Korendyke, C. M., Paswaters, S. E., Wang, D., Schwenn, R., Lamy, P., Llebaria, A., and Bout, M. V.: LASCO Observations of Disconnected Magnetic Structures Out to Beyond 28 Solar Radii During Coronal Mass Ejections, Sol. Phys., 175, 685-698, doi: 10.1023/A:1004994214697, 1997.

Srivastava, N., Schwenn, R., Inhester, B., Martin, S. F., and Hanaoka, Y.: Factors Related to the Origin of a Gradual Coronal Mass Ejection Associated with an Eruptive Prominence on 2122 June 1998, Astrophys. J., 534, 468-481, doi:10.1086/308749, 2000.

Sterling, A. C. and Moore, R. L.: External and Internal Reconnection in Two Filament-Carrying Magnetic Cavity Solar Eruptions, Astrophys. J., 613, 1221-1232, 2004.

Švestka, Z.: Varieties of Coronal Mass Ejections and Their Relation to Flares, Space Sci. Rev., 95, 135-146, 2001.

Temmer, M., Veronig, A. M., Vršnak, B., and Miklenic, C.: Energy Release Rates along $\mathrm{H} \alpha$ Flare Ribbons and the Location of Hard X-Ray Sources, Astrophys. J., 654, 665-674, doi: 10.1086/509634, 2007a.

Temmer, M., Veronig, A. M., Vršnak, B., Rybák, J., Gömöry, P., Stoiser, S., and Maričić, D.: Acceleration in Fast Halo CMEs and synchronized flare HXR bursts, Astrophys. J. Lett., in press, 2007 b.

Titov, V. S. and Démoulin, P.: Basic topology of twisted magnetic configurations in solar flares, Astron. Astrophys., 351, 707-720, 1999.

Török, T. and Kliem, B.: Confined and Ejective Eruptions of Kinkunstable Flux Ropes, Astrophys. J. Lett., 630, L97-L100, doi: 10.1086/462412, 2005.

Van der Holst, B., Jacobs, C., and Poedts, S.: Simulation of a Breakout Coronal Mass Ejection in the Solar Wind, Astron. Astrophys., 671, L77L80, 2007.

van Tend, W. and Kuperus, M.: The development of coronal electric current systems in active regions and their relation to filaments and flares, Sol. Phys., 59, 115-127, 1978.

Veronig, A. M., Karlický, M., Vršnak, B., Temmer, M., Magdalenić, J., Dennis, B. R., Otruba, W., and Pötzi, W.: X-ray sources and magnetic reconnection in the X3.9 flare of 2003 November 3, Astron. Astrophys., 446, 675-690, doi:10.1051/ 0004-6361:20053112, 2006.

Vršnak, B.: The oscillating loop prominence of July 17, 1981, Sol. Phys., 94, 289-297, 1984.

Vršnak, B.: Eruptive instability of cylindrical prominences, Sol. Phys., 129, 295-312, 1990.

Vršnak, B.: Dynamics of solar coronal eruptions, J. Geophys. Res., 106, 25 249-25 260, doi:10.1029/2000JA004007, 2001.

Vršnak, B. and Skender, M.: 2 1/2-Dimensional Reconnection Model and Energy Release in Solar Flares, Sol. Phys., 226, $97-$ 119, doi:10.1007/s11207-005-5701-3, 2005.

Vršnak, B., Ruždjak, V., Messerotti, M., Mouradian, Z., and Urbarz,
H.: Reconnection driven by an erupting filament in the May 14, 1981 flare, Sol. Phys., 114, 289-310, 1987.

Vršnak, B., Ruždjak, V., Brajša, R., and Zloch, F.: Oscillatory motions in an active prominence, Sol. Phys., 127, 119-128, 1990.

Vršnak, B., Ruzdjak, V., and Rompolt, B.: Stability of prominences exposing helical-like patterns, Sol. Phys., 136, 151-167, 1991.

Vršnak, B., Magdalenić, J., Aurass, H., and Mann, G.: Bandsplitting of coronal and interplanetary type II bursts. II. Coronal magnetic field and Alfvén velocity, Astron. Astrophys., 396, 673-682, doi:10.1051/0004-6361:20021413, 2002.

Vršnak, B., Warmuth, A., Maričić, D., Otruba, W., and Ruždjak, V.: Interaction of an Erupting Filament with the Ambient Magnetoplasma and Escape of Electron Beams, Sol. Phys., 217, 187-198, 2003.

Vršnak, B., Maričić, D., Stanger, A. L., and Veronig, A.: Coronal Mass Ejection of 15 May 2001: II. Coupling of the CME Acceleration and the Flare Energy Release, Sol. Phys., 225, 355-378, doi:10.1007/s11207-004-4995-x, 2004a.

Vršnak, B., Ruždjak, D., Sudar, D., and Gopalswamy, N.: Kinematics of coronal mass ejections between 2 and 30 solar radii. What can be learned about forces governing the eruption?, Astron. Astrophys., 423, 717-728, doi:10.1051/0004-6361:20047169, 2004b.

Vršnak, B., Sudar, D., and Ruždjak, D.: The CME-flare relationship: Are there really two types of CMEs?, Astron. Astrophys., 435, 1149-1157, doi:10.1051/0004-6361:20042166, 2005.

Vršnak, B., Maričić, D., Stanger, A. L., Veronig, A. M., Temmer, M., and Roša, D.: Acceleration Phase of Coronal Mass Ejections: I. Temporal and Spatial Scales, Sol. Phys., 241, 85-98, doi:10.1007/s11207-006-0290-3, 2007.

Vršnak, B., Ruzdjak, V., Rompolt, B., Rosa, D., and Zlobec, P.: Kinematics and evolution of twist in the eruptive prominence of August 18, 1980, Sol. Phys., 146, 147-162, 1993.

Vršnak, B., Klein, K.-L., Warmuth, A., Otruba, W., and Skender, M.: Vertical Dynamics of the Energy Release Process in a Simple two-Ribbon Flare, Sol. Phys., 214, 325-338, 2003.

Wang, H., Qiu, J., Jing, J., and Zhang, H.: Study of Ribbon Separation of a Flare Associated with a Quiescent Filament Eruption, Astrophys. J., 593, 564-570, doi:10.1086/376360, 2003.

Wang, Y.-M. and Sheeley, Jr., N. R.: Observations of Core Fallback during Coronal Mass Ejections, Astrophys. J., 567, 1211-1224, doi:10.1086/338757, 2002.

Wang, Y.-M., Sheeley, N. R., Howard, R. A., St. Cyr, O. C., and Simnett, G. M.: Coronagraph observations of inflows during high solar activity, Geophys. Res. Lett., 26, 1203-1206, doi: 10.1029/1999GL900209, 1999.

Webb, D. F. and Cliver, E. W.: Evidence for magnetic disconnection of mass ejections in the corona, J. Geophys. Res., 100, 5853$5870,1995$.

Webb, D. F., Burkepile, J., Forbes, T. G., and Riley, P.: Observational evidence of new current sheets trailing coronal mass ejections, J. Geophys. Res. (Space Physics), 108, SSH6-1-123, doi: 10.1029/2003JA009923, 2003.

Williams, D. R., Török, T., Démoulin, P., van Driel-Gesztelyi, L., and Kliem, B.: Eruption of a Kink-unstable Filament in NOAA Active Region 10696, Astrophys. J. Lett., 628, L163-L166, doi: 10.1086/432910, 2005.

Wood, B. E., Karovska, M., Chen, J., Brueckner, G. E., Cook, J. W., and Howard, R. A.: Comparison of Two Coronal Mass 
Ejections Observed by EIT and LASCO with a Model of an Erupting Magnetic Flux Rope, Astrophys. J., 512, 484-495, doi: 10.1086/306758, 1999.

Wu, S. T., Andrews, M. D., and Plunkett, S. P.: Numerical Magnetohydrodynamic (MHD) Modeling of Coronal Mass Ejections (CMEs), Space Sci. Rev., 95, 191-213, 2001.

Yashiro, S., Gopalswamy, N., Michalek, G., St. Cyr, O. C., Plunkett, S. P., Rich, N. B., and Howard, R. A.: A catalog of white light coronal mass ejections observed by the SOHO spacecraft, J. Geophys. Res. (Space Physics), 109, A077105, 1-11, doi: 10.1029/2003JA010282, 2004.

Yokoyama, T., Akita, K., Morimoto, T., Inoue, K., and Newmark, J.: Clear Evidence of Reconnection Inflow of a Solar Flare, Astrophys. J. Lett., 546, L69-L72, doi:10.1086/318053, 2001.

Zhang, J.: A Study on the Acceleration of Coronal Mass Ejections, in: Coronal and Stellar Mass Ejections, edited by Dere, K., Wang, J., and Yan, Y., vol. 226 of IAU Symposium, 65-70, 2005 .
Zhang, J. and Dere, K. P.: A Statistical Study of Main and Residual Accelerations of Coronal Mass Ejections, Astrophys. J., 649, 1100-1109, doi:10.1086/506903, 2006.

Zhang, J., Dere, K. P., Howard, R. A., Kundu, M. R., and White, S. M.: On the Temporal Relationship between Coronal Mass Ejections and Flares, Astrophys. J., 559, 452-462, doi:10.1086/ 322405, 2001.

Zhang, J., Dere, K. P., Howard, R. A., and Vourlidas, A.: A Study of the Kinematic Evolution of Coronal Mass Ejections, Astrophys. J., 604, 420-432, doi:10.1086/381725, 2004.

Zhang, M. and Low, B. C.: The Hydromagnetic Nature of Solar Coronal Mass Ejections, Ann. Rev. Astron. Astrophys., 43, 103 137, 2005.

Žic, T., Vršnak, B., and Skender, M.: The Magnetic Flux and SelfInductivity of a Thick Toroidal Current, J. Plasma Phys., 73, 741-756, doi:doi:10.1017/S0022377806006209, 2007. 